\title{
Ärztliche Zeugnisse auf dem medizinischen Markt. Professionalisierte Zeugenschaft im wachsenden Verwaltungsapparat des frühen 19. Jahrhunderts
}

Lina Gafner

\section{Summary}

This article deals with medical certificates issued by the physician Cäsar Adolf Bloesch (1804-1863) from Biel/Bienne. It aims at examining this service as a part of the medical market in the early nineteenth century. Firstly, it focuses on four administrative procedures which show how the standardized medical certificate was established at the time. Secondly, it examines how expert testimony in general and medical testimony in particular depended on public and private demand. Finally, this study argues that by attesting health or illness, physicians could gain their patients' confidence and increase their own social capital. For patients a medical certificate could imply release from moral judgement.

Keywords: medical certificate, medical market, testimony, administration

\section{Zusammenfassung}

Der Artikel befasst sich mit den ärztlichen Zeugnissen des Bieler Arztes Cäsar Adolf Bloesch (1804-1863), mit dem Ziel, diese Dienstleistung als Teil des medizinischen Marktes im frühen 19. Jahrhundert zu beleuchten. Zuerst wird anhand von vier administrativen Verfahren gezeigt, wie sich das genormte schriftliche Zeugnis in dieser Zeit durchgesetzt hat. In einem weiteren Schritt wird die Zeugenschaft durch Sachverständige im Allgemeinen und durch Ärzte im Speziellen im Zusammenhang mit staatlicher und privater Nachfrage betrachtet. Schließlich wird die These aufgestellt,

Lina Gafner, Institut für Medizingeschichte, Bühlstraße 26, CH-3012 Bern (lina.gafner@img. unibe.ch). 
dass für die Ärzte das Potenzial der Zeugnisse darin lag, Klienten an sich zu binden und ihr soziales Kapital zu vermehren. Für Patientinnen und Patienten wiederum konnte die ärztliche Zeugenschaft eine Befreiung von moralischen Urteilen bedeuten.

Schlagworte: ärztliche Zeugnisse, medizinischer Markt, Zeugenschaft, Administration

Maria Wegmann kannte den Arzt Cäsar Adolf Bloesch bereits, als sie am 30. März 1839 bei ihm für ein Zeugnis vorstellig wurde. ${ }^{1}$ Er hatte sie und ihren Mann vier Jahre zuvor erstmals behandelt. Unterdessen war ihr Mann verstorben, und sie lebte bei der Familie ihrer Tochter in ärmlichen Verhältnissen. Die Frau litt seit Jahren unter rheumatischen Schmerzen, und nun wollte die Landsaßenkommission, die im Kanton Bern für die Heimatlosen zuständig war, ${ }^{2}$ ihren jährlichen Beitrag kürzen. In seinem Zeugnis empfahl Bloesch der Landsaßenkommission, von dieser Kürzung abzusehen, weil Maria Wegmann «verdienstunfähig» sei und auch ihr Schwiegersohn nicht für sie sorgen könne. Das Zeugnis stellte er derselben Kommission in Rechnung. ${ }^{3}$

Die Landstadt Biel, die 1798 mit ihren Nachbargemeinden als «canton de Bienne» von Frankreich annektiert worden war, fiel nach der Niederlage Napoleons 1814 an ihre «alte Bundesgenossin» ${ }^{4}$ Bern. Mit dem Sieg der liberalen Opposition in Bern 1831 wurde Biel Teil des Demokratisierungsund Zentralisierungsprozesses im Kanton, dem Mitte des 19. Jahrhunderts ein Verwaltungsapparat entwuchs, der Gemeinden, Beamten und Bürgern vermehrte Schreibarbeit abforderte. So gehörte zu den alltäglichen Tätigkeiten des Bieler Arztes Bloesch (1804-1863) nicht nur die «Herstellung des gesunden Zustandes $»^{5}$ seiner kranken Klientel, sondern zunehmend auch das Verfassen von Gutachten, Berichten und Zeugnissen. Die ärztlichen

1 Diese Arbeit ist im Rahmen des vom Schweizerischen Nationalfonds unterstützten Forschungsprojekts «Die Praxis des Bieler Arztes Cäsar Adolf Bloesch (1804-1863)» entstanden.

2 Meier/Wolfensberger 1998, 374-381.

3 «Für Landsassencommission ärztliches Zeugnis: Maria Wegmann, Peters Wittwe, über 60 Jahre alt, seit 20 Jahren an Rheumatismen leidend, daher schon oft, und zwar auch im Laufe des letzten Jahres in ärztlicher Behandlung, seit Langem an arthritischen Magenschmerzen leidend, sowohl ihres Alters als dieser ihrer körperlichen Gebrechen wegen verdienstunfähig, wohnt bei ihrem Tochtermanne Wehren zu Biel, dem deren Unterhalt gänzlich zur Last fällt, während er selbst als Taglöhner kaum für seinen Unterhalt zu sorgen im Stande ist, so dass wohl schwerlich eine Reduction der jährlichen Steuer von 50 Fr zulässig sein möchte.» StadtABiel, 4 A 1, Tagebuch, 30.3.1839, Bd. 15, 125.

4 Bloesch 1853, VI.

5 Bloesch 1866, 122. 
Zeugnisse, die Bloesch für seine Patientinnen und Patienten verfasst und gleichzeitig als Abschrift in seinem Praxisjournal hinterlassen hat, sind aber nicht bloß als Produkt der zunehmenden Formalisierung zu lesen. Sie belegen vielmehr ebenso die Inanspruchnahme einer sich etablierenden ärztlichen Dienstleistung durch die Bevölkerung. Welche Rolle die Zeugnisse auf dem medizinischen Markt des frühen 19. Jahrhunderts gespielt haben, wird im Folgenden am Beispiel der von Bloesch verfassten Zeugnisse genauer ausgeführt.

\section{Vielfältige Nachfrage und Normierung}

Die wesentliche Gemeinsamkeit der ärztlichen Zeugnisse liegt darin, dass sie jeweils auf eine Person ausgestellt und auch von dieser Person oder ihren Angehörigen in Auftrag gegeben wurden. Formal waren die Zeugnisse an diese Person gebunden, das heißt, dass dem ärztlichen Urteil mindestens deren Name und meistens auch deren Heimatort vorausgingen. Inhaltlich lassen die Atteste dagegen auf eine Vielfalt an Verwendungszwecken schließen, wie die folgenden Beispiele veranschaulichen.

Beispiel 1: «Für Gottlieb Dutoit, ärztliches Zeugniss: Gottlieb Dutoit, Soldat bei der Scharfschützencompagnie, geb. 1806 zu Nidau, Stammquartier Nidau, und wohnhaft zu Nidau, ist mit Blutspeien, Engbrüstigkeit und Herzklopfen schon seit längerer Zeit behaftet, und daher zu persönlicher Leistung des Militärdiensts bei der Frühlingsmusterung des Jahres untauglich.» ${ }^{6}$ Abraham Gottlieb Dutoit, Müller in Nidau und Sohn des dortigen Bürgermeisters und seit 1832 bei Bloesch in Behandlung, suchte den Arzt ab 1834 mehrmals auf, um sich vom Militärdienst freischreiben zu lassen. Die Dutoits gehörten zur treuen Klientel Bloeschs, und das blieb auch so, nachdem Abraham Dutoit 1841 36-jährig an seinem Leiden gestorben war.

Beispiel 2: «Für Moser-Weienet ärztliches Zeugnis: Der Unterzeichnete bescheint:1) dass Elisabeth Moser, geb. Weienet, von Madretsch, am 29. Sept. Nachmittags hieher nach Biel gekommen sei, um sich einer am Morgen desselben Tages erlittenen Misshandlung wegen ärztlich untersuchen zu lassen; 2) dass bei dieser Untersuchung sich erfunden habe, dass Elisabeth Moser-Weienet ungefähr in der Mitte einer Schwangerschaft sich befinde, und dermassen gröblich misshandelt, geschlagen oder mit Füssen auf die Geschlechtstheile getreten worden sei [...]. In wie fern diese Misshandlung auf Leben und Gesundheit der Leibesfrucht Einfluss haben könnte, ist

6 StadtABiel, 4 A 1, Tagebuch, 2.4.1840, Bd. 17, 182. 
gegenwärtig nicht zu bestimmen.» ${ }^{7}$ Elisabeth Moser-Weienet kam unter wahrscheinlich großen Schmerzen gleich am Tag ihrer Misshandlung ${ }^{8}$ nach Biel zu Bloesch. Der Arzt sollte bestätigen, dass eine allfällige Fehlgeburt auf die erlittene Gewalttätigkeit zurückzuführen sei und nicht auf eine Abtreibung, um die Schwangere so gegen eine mögliche Anklage abzusichern.

Beispiel 3: «Für Bendicht Wälte, ärztliches Zeugnis: Bendicht Wälte von Sutz, Schuhmacher, in der Lehre zu Sutz, ist mit keiner Hautkrankheit behaftet, hat die Narben ächter Schutzpoken am Arme so dass ihm ein Wanderbuch ausgestellt werden kann.» ${ }^{9}$ Nicht nur Krankheit lässt sich bescheinigen, sondern auch Gesundheit. Bendicht Wälti ist nur dieses eine Mal bei Bloesch registriert.

Beispiel 4: «Für H. Widmer, ärztliches Zeugnis: der Unterzeichnete bescheint (richtig?), dass er den H. Widmer, Wirth zur Krone in Biel, seit dem 10. Mai besucht und ärztlich versorgt habe; dass $\mathrm{H}$. Widmer an dem delirium tremens gelitten, welches aber allmählich bei dem Gebrauche des opium verschwunden sei; [...] dass H. Widmer wieder bei ungestörtem Gebrauche seiner Geisteskräfte sich befinde, und dass die Bevogtung aufgehoben werden könne.» ${ }^{10}$ Jakob Widmer war seit drei Jahren in Behandlung bei Bloesch, der bei ihm Anzeichen von Geistesschwäche registrierte, die allerdings manchmal fast ganz verschwanden, um dann wiederum stärker zu werden. Widmer hatte auch schon einen Anstaltsaufenthalt hinter sich und wurde unterdessen bevormundet. Um diese «Bevogtung» aufheben zu lassen, nahm er wiederholt Bloeschs Referenz in Anspruch.

Die Nachfrage nach ärztlichen Zeugnissen ist zumindest zum Teil auf Vorschriften zurückzuführen, die solche Belege verlangten. In den bernischen Gesetzen und Verordnungen taucht diese Anforderung ab der Wende zum 19. Jahrhundert zunehmend auf. Beispielsweise wurde jemand, der nicht aus eigener Kraft die Heimatgemeinde erreichen konnte und deshalb eine Armenfuhre benötigte, 1811 per Verordnung dazu aufgefordert, «nöthig findenden Falls ein ärztliches Zeugniss» einzuholen. ${ }^{11}$ Ab 1848 mussten Arbeitsunfähige und unheilbar Kranke, für die keine Verwandten sorgen konnten, ihre Unheilbarkeit durch einen Arzt bescheinigen lassen, um von

7 StadtABiel, 4 A 1, Tagebuch, 9.10.1837, Bd.12,1.

8 Bloesch bezeichnet jede körperliche Gewalt gegen eine Person als Misshandlung, auch wenn mehrere Personen in eine Schlägerei verwickelt waren. Diese Bedeutung, die keinen sexuellen Gehalt trägt, wird im Folgenden beibehalten.

9 StadtABiel, 4 A 1, Tagebuch, 13.3.1842, Bd. 21, 53.

10 StadtABiel, 4 A 1, Tagebuch, 13.8. 1847, Bd. 30, 203.

11 Gesetze, Dekrete und Verordnungen des Kantons Bern, 4 (1813), 110. 
der neuen Spendenanstalt für Unheilbare unterstützt zu werden. ${ }^{12}$ Und mittellose Jünglinge erhielten ab 1848 nur dann ein Stipendium, um ein Handwerk zu erlernen, wenn sie neben einem Leumunds- und einem Armutszeugnis auch ein ärztliches Zeugnis vorweisen konnten, das ihre Tüchtigkeit belegte. ${ }^{13}$ Für all diese Verordnungen finden sich auch entsprechende Beispiele unter den von Bloesch verfassten Zeugnissen. Besonders gefragt waren aber Armutszeugnisse, Militärdienstzeugnisse, Atteste bei einer Misshandlung und solche, die Gesundheit bescheinigten.

\section{Arbeitsunfähigkeit und Armut}

Die vormoderne gemeindliche Armenfürsorge baute auf eine moralische Unterscheidung zwischen unterstützungswürdigen und selbstverschuldeten Armen. Wem selbstverschuldete Armut unterstellt wurde, der besaß keine Aussicht auf Armengenössigkeit. ${ }^{14}$ Von Amts wegen waren in der alten Republik Bern die Landvögte dafür zuständig, die Armutsumstände einer Person oder Familie nachzuweisen und sie als unterstützungswürdig zu befinden. ${ }^{15}$ Allerdings ist anzunehmen, dass die Verfahrenspraxis weitaus komplexer aussah. Norbert Finzsch hat für Köln nachgewiesen, dass um 1800 nebst den hauptsächlich zuständigen Bürgerhauptleuten auch Nachbarn oder andere Bürger der Stadt schriftliche Zeugnisse ausgestellt haben oder auch mündlich bezeugten, dass eine Person oder Familie Armenhilfe benötigte. Sei dies aus Nächstenliebe oder aber weil Nachbarn lieber die öffentliche Hand bezahlen ließen als selbst in die Tasche zu greifen. Einzelne Bürger fielen als besonders engagierte Zeugen auf, deren Bereitschaft sich auch herumzusprechen schien. Ärzte stellten dabei eine kleine Minderheit dar, obwohl in vielen Fällen Krankheit mit der Armut zusammenhing. ${ }^{16}$ Klare Kriterien für die Beurteilung von Armut fehlten. Das Nebeneinander verschiedener Begründungsmuster ${ }^{17}$ war allerdings Ausdruck einer Übergangsphase, und die Forderung nach einem einheitlichen, transparenten Vorgehen erstarkte in Köln zunehmend. Dass zur selben Zeit auch in Bern das gängige Verfahren eine aufseiten der Behörden unerwünschte Dynamik angenommen hatte, lässt eine Verordnung von 1803 erahnen: «Die Herren

12 Gesetze, Dekrete und Verordnungen des Kantons Bern, 3 (1848), 72.

13 Gesetze, Dekrete und Verordnungen des Kantons Bern, 3 (1848), 192.

14 Für einen Überblick vgl. Ludi/Matter/Rietmann 2011,192-204. Zwei publizierte Lizentiatsarbeiten widmen sich der Armenfürsorge in einer Berner Gemeinde unter mikrohistorischer Perspektive: Schläpfer 2002 und Baumer 2002.

15 Flückiger Strebel 2002, 48.

16 Finzsch 1990, 55-86.

17 Finzsch führt das Beispiel eines Arztes an, der soziale Ursachen für die Armut geltend machte, und zeigt, dass Pfarrer dagegen fast immer moralische Gründe sprechen ließen und das Almosenideal hochhielten. Finzsch 1990, 76. 
Pfarrer und Behörden auf dem Lande, welche im Fall sind, Armuthszeugnisse auszustellen, sollen selbige verschlossen ausstellen, da von diesen offenen Empfehlungen häufiger Missbrauch ist gemacht worden.» In einem Kreisschreiben von 1821 mussten die Adressaten auf dem Land erneut an diese Vorschrift erinnert werden. ${ }^{18}$

Bevölkerungswachstum, Arbeitsmigration und die Folgen der Agrarmodernisierung führten in der ersten Hälfte des 19. Jahrhunderts zu einer Massenarmut, der viele Gemeinden nicht mehr gewachsen waren. Die Zahl der Bedürftigen nahm stetig zu, während im Zuge der liberalen Reformen die Fürsorgepflicht der Gemeinden gleichzeitig relativiert und die Armensteuer vielerorts abgeschafft wurden. Das hatte zur Folge, dass die Kriterien für das Recht auf Unterstützung immer strenger wurden. ${ }^{19}$ Die Armenkommission der Burgergemeinde Biel schöpfte für die Armenfürsorge aus dem Armengut Tischgeld und Pensionen und aus dem Spitalgut Unterstützung im Krankheitsfall. Die Bedürftigen erschienen jeweils persönlich vor der Kommission und suchten um Hilfe an, indem sie den Grund ihrer Not darlegten. Schriftliche Gesuche wurden nicht behandelt. Die acht Kommissionsmitglieder entschieden gemeinsam darüber, wer welche Hilfe erhalten sollte, und ein ausführender Armenverwalter prüfte darüber hinaus die Verhältnisse bei den Bedürftigen zu Hause. Unterstützt wurden vornehmlich Kinder, kranke Menschen, deren Angehörige, Witwen und geschiedene Frauen. Die Hilfe deckte neben dem Bedarf an Holz oftmals Arzt- und Apothekerkosten, Kosten für Badekuren, Reisekosten in die Kurbäder, und sie sicherte die Verköstigung der zurückbleibenden Familien oder entschädigte pflegende Verwandte. ${ }^{20}$ Wer um Armenhilfe ansuchen wollte, war lange Zeit auf den Beleg angewiesen, dass keine moralische Fehlbarkeit hinter der Bedürftigkeit stand, was vor allem Frauen betraf. ${ }^{21}$ Der liberale und konservative Konsens der Regenerationsära, demgemäß ein arbeitsfähiger Mensch sich aus eigener Kraft erhalten müsse, ${ }^{22}$ fragte dagegen in erster Linie nach einem Beleg für die vorübergehende oder dauernde Arbeitsunfähigkeit einer Person. Entsprechende Arztzeugnisse entwickelten sich so zu einer Grundlage der anerkannten Bedürftigkeit, und der Arzt wurde zum wichtigsten Zeugen für Armut.

18 Gesetze, Dekrete und Verordnungen des Kantons Bern, 1 (1805), 156f., und 2 (1821), 310.

19 Vgl. Ludi 1975.

20 Vgl. StadtABiel, BG-Biel, 226: Armengutsrechnungen und Helfer 1994, 57-60 und 68-70.

21 Vgl. Ludi 1989.

22 Vgl. Ludi 1975. 


\section{Militärdienst}

Der obligatorische Militärdienst war und ist ein Zwang, dem sich zumindest der für diese Form des Staatsdiensts nicht begeisterungsfähige Teil der betroffenen männlichen Bevölkerung seit dessen Bestehen mit allen möglichen Mitteln zu entziehen versucht. Interventionen des Pfarrers oder die Bestechung der mit der Aushebung betrauten Beamten standen bei den Bemühungen der jungen Männer oder ihrer Eltern zuerst gleichberechtigt neben dem sich bald durchsetzenden Mittel des Arztzeugnisses. Um auch bei fehlender Krankheit an ein solches zu gelangen, mussten sie entweder den Arzt bestechen oder aber ein Leiden simulieren, dessen eindeutige Feststellung schwierig war. ${ }^{23}$ Im Kampf gegen erkaufte Arztzeugnisse wurden vielerorts besser kontrollierbare Militärärzte eingeführt, die sich allerdings auch nicht als unbestechlich erwiesen. ${ }^{24}$ Bern führte 1818 mit der «neuen Militär-Verfassung des Cantons» die allgemeine Wehrpflicht ein und definierte 1826 nebst den Straffälligen all jene als Untüchtige, die «durch Attestate der dazu bestellten Kreis-Aerzte bescheinigen können, dass sie durch körperliche Gebrechen oder schlechten Gesundheitszustand zum Militairdienst untauglich sind». ${ }^{25} 1835$ wurden die Kriterien präzisiert und verschärft. Um von der Dienstpflicht befreit zu werden, war nun «die Eingabe eines vom Kreiskommandanten und zwei Kreisärzten ausgefertigten Befundscheines nöthig». ${ }^{26}$ Die zur Ausstellung von Untauglichkeitszeugnissen berechtigten Personen beschränkten sich also per bernische Gesetzgebung auf die durch die Sanitätskommission gewählten, vereidigten Kreisärzte. ${ }^{27}$

Der Nachlass Bloesch beweist allerdings, dass auch im Bereich des Militärs die Verfahrenspraxis von der Gesetzgebung abwich. Bloesch löste im Mai 1838 seinen Kollegen Charles Théodore Schaffter als Kreisarzt in Biel ab und besetzte diese Funktion bis zum August 1848. ${ }^{28}$ Obwohl er nach dem Gesetz nur in dieser Zeit die Untauglichkeit von Wehrdienstpflichtigen hätte nachweisen dürfen, finden sich in seinem Journal zahlreiche Zeugnisse, die Bloesch sowohl vor als auch nach dieser Zuständigkeit ausgestellt hat, was auch auf den in Beispiel 1 erwähnten Abraham Dutoit zutrifft.

23 Maren Lorenz verweist auf die Epilepsie als beliebtes Vehikel, vgl. Lorenz 1999, 398. Vgl. a. Frevert 2001, 71-81.

24 Für Preußen vgl. Frevert 2001, 73.

25 Gesetze, Dekrete und Verordnungen des Kantons Bern, 2 (1821), 28, und 4 (1828), 194.

26 Gesetze, Dekrete und Verordnungen des Kantons Bern, 5 (1835), 232.

27 StAB, BB II 546, Rapporte über Beeidigung von Kreis- und Bataillonsärzten, 1832-1835.

28 StAB, BB II 508, Kontrolle über die Ernennung der Militärbeamten in den 8 Kreisen, 1832-1853. 


\section{Misshandlung}

Die Zuständigkeit für die gerichtliche Wundbegutachtung verschob sich seit dem späten 16. Jahrhundert von den Händen der Wundärzte in diejenigen der akademischen Mediziner. ${ }^{29}$ Den Bieler Gerichtsakten, die sich mit strafbaren Handlungen gegen Personen befassen, ist pro Fall mindestens ein Zeugnis eines behandelnden Arztes oder Wundarztes beigelegt, der oft schon kurz nach dem Vorfall aufgesucht oder gerufen worden war. Sie sind von den Gerichtsgutachten zu unterscheiden, die das zuständige Amt in schwierigen Fällen selbst in Auftrag gab. ${ }^{30}$ Die Praxis, nach einer erlittenen Misshandlung einen Fachmann aufzusuchen und die Verletzungen von ihm bezeugen und in ihrer Schwere einschätzen zu lassen, scheint verbreitet gewesen zu sein. Die Zeugnisse erzählen von Schlägereien zwischen Männern, meist Gesellen, von Überfällen auf der Landstraße oder von Verletzungen, deren ungenannter Urheber im Familienkreis zu vermuten ist. Frauen stellen mit etwa 17\% die deutliche Minderheit jener Misshandelten dar, denen Bloesch ein Zeugnis ausstellte. ${ }^{31}$ Dem Namen und dem Heimatort folgen in diesen Zeugnissen der Zeitpunkt und die Umstände der Untersuchung, manchmal geben sie auch Äußerungen der verletzten Person wieder, bevor eine genaue Beschreibung der Verletzung und die Einschätzung ihrer Tragweite das Dokument abschließen. Während sich Bloesch relativ genau an die formalen Anforderungen hielt, wurden andere Ärzte durch die Verwaltung zurechtgewiesen, wie im Falle des aus Württemberg stammenden Arztes Bernhard Karl Finkbeiner, der im nahe Biel gelegenen Mett praktizierte. Finkbeiner erhielt im Juni 1836 ein Schreiben des Gerichtspräsidenten von Biel, Alexander Schöni, worin dieser von Finkbeiner die Neuschrift eines wenige Tage zuvor verfassten Zeugnisses verlangte, das Finkbeiner einem in einer Schlägerei verletzten Mann ausgestellt hatte. Dem Dokument, so Schöni, fehlten die entscheidenden Teile, nämlich ein Urteil darüber, «ob und wie lange derselbe dadurch verhindert sein möchte, seine gewöhnlichen Arbeiten zu verrichten, so wie auch, wie viel Tage, Sie glauben, dass erforderlich seÿen, um denselben von seinen erhaltenen Wunden völlig wieder herzustellen». ${ }^{32}$ Tatsächlich ist jenen ärztlichen Zeugnissen, die für das Gericht oder die Armenhilfe bestimmt waren, immer eine detaillierte Prognose zu entnehmen, die ein Gerichtsurteil oder auch den Umfang der Armenhilfe entscheidend beeinflussen konnte. Solche Prognosen ließen beispielsweise vermuten, «dass man den Kranken nicht als außer Gefahr betrachten könne,

29 Vgl. Fischer-Homberger 1983, 43-52.

30 Zum Aufzeichnungssystem der gerichtsärztlichen Gutachten vgl. Fangerau/Müller 2010.

31 Dies ergaben die statistischen Auswertungen des Krankenjournals von C. A. Bloesch.

32 StAB, Bez Biel B 825. 
weil die Heftigkeit des erhaltenen Schlages innere Verletzungen möglicher Weise zur Folge haben könnte, welche noch ein schlimmes Ende nehmen dürften», oder sagten voraus, dass «die Wunde des Christian Pfaute schwerlich ohne Entzündung, vielleicht nicht ohne Eyterung heilen [wird], was die Herstellung bis auf den 14. Tag verzögern möchte».33

\section{Gesundheitsnachweis}

Unter jenen Zeugnissen, die Gesundheit attestierten, sind einige auf Frauen ausgestellt, die eine Anstellung suchten oder einen Beruf lernen wollten. Bloesch bescheinigte Anna Maria Sager, dass sie die Arbeiten einer Dienstmagd «ohne Nachtheil für ihre Gesundheit» verrichten könne, ${ }^{34}$ er beurteilte Anna Lechot trotz der «Steifheit dreier Finger» als für den Beruf der Hebamme geeignet, ${ }^{35}$ und für Rosette Mühlheims Aufnahme ins Lehrerseminar sah er kein Hindernis, da diese von «gesunder und kräftiger Körperbeschaffenheit» sei. ${ }^{36}$ Auch Erziehungsanstalten und andere Heime, wie sie in der Zeit zahlreich gegründet wurden, verlangten das Attest eines Arztes. Der dreizehnjährige Abraham Kunz sei seit seiner Geburt blind, sonst aber «mit keinem Uebel behaftet», weshalb er ins Blindenheim aufgenommen werden könne. ${ }^{37}$ Den fünfzehnjährigen Eduard Christen, dessen körperliche und geistige Entwicklung «auf einer etwas tiefen Stuffe» geblieben sei, empfahl Bloesch für die Überweisung in eine Anstalt, da er die «ächten Schutzpocken» bereits gehabt habe und deshalb keine Ansteckungsgefahr bestehe ${ }^{38}$ Die Angst vor den Pocken liegt einer großen Zahl von Zeugnissen zugrunde, die von der überstandenen Krankheit oder der vollzogenen Impfung erzählen. Gesellen und andere im Aufbruch Begriffene sprachen für solch eine Bestätigung vor, weil sie ohne diese keine Pässe und Wanderbücher erhielten.

Das sich wandelnde Medizinal- und Fürsorgewesen brachte nicht nur neue Anstalten und vermehrte gesundheitspolitische Maßnahmen hervor, sondern auch sogenannte «gegenseitige Hülfsgesellschaften», die Vorläufer der heutigen Krankenkassen und Nachfolger der Fürsorge durch Zünfte und Gesellenverbände. ${ }^{39} \mathrm{Um}$ die Gründung solcher Gesellschaften voranzutreiben und diese als bleibende Institutionen zu etablieren, gab die Schweizerische Statistische Gesellschaft 1867 eine Sammlung von Grundsätzen

33 StadtABiel, 4 A 1, Tagebuch, 4.8.1834, Bd. 6, 102 und 19.8.1834, Bd. 6, 117.

34 StadtABiel, 4 A 1, Tagebuch, 23.8.1834, Bd. 6, 126.

35 StadtABiel, 4 A 1, Tagebuch, 2.11.1856, Bd. 44, 443.

36 StadtABiel, 4 A 1, Tagebuch, 1.9.1844, Bd. 25, 217.

37 StadtABiel, 4 A 1, Tagebuch, 16.7.1841, Bd. 19, 408.

38 StadtABiel, 4 A 1, Tagebuch, 11.6.1851, Bd. 36, 227.

39 Vgl. hierzu Fröhlich 1976, Gruner 1968, 1000-1024, und Reusser 1985. 
heraus, deren Beachtung sie den Kassen empfahl. Ganz oben auf der Liste stand das Prinzip: «Jeder Aufzunehmende muss im Zeitpunkt der Anmeldung gesund sein, was nöthigenfalls durch ein Zeugniss eines patentirten Arztes zu bescheinigen ist.» ${ }^{40}$ Der Blick in die Statuten bereits früher gegründeter Kassen zeigt eine diesbezüglich uneinheitliche Praxis. Die «Kranken- und Hilfskassa» des Stadtbezirks Biel schränkte 1838 ihre potenziellen Mitglieder auf solche ein, die «gesund und ohne bedeutende körperliche Gebrechen» seien, ohne hierfür einen Beleg zu verlangen. Anders die Statuten der bernischen Lehrerkasse von 1858: «Wer die Aufnahme in die Kasse verlangt, hat die Meldung bei dem Bezirksvorsteher zu besorgen und demselben einzusenden: [...] eine ärztliche Bescheinigung dass er mit keinem Übel behaftet sei, das ihn an der längern Ausübung seines Berufes zu verhindern drohe. ${ }^{41}$ Eine voranschreitende Formalisierung dieser Institutionen ist nicht zu übersehen, und die Pflicht, die eigene Gesundheit nachzuweisen, scheint sich rasch verbreitet zu haben.

\section{Experten als Zeugen}

Etymologisch verweist der Begriff des Zeugnisses auf die gerichtliche Praxis der Zeugenschaft. Die Glaubwürdigkeit und Unabhängigkeit der Zeugen wurde in der Frühen Neuzeit zum zentralen Anliegen des Justizwesens, wie Barbara Shapiro am Beispiel Englands aufzeigt. Der gute Ruf, das Geschlecht, Besitz, Bildung und Alter machten letztlich einen vertrauenswürdigen Zeugen aus. ${ }^{42}$ Medizinische Atteste stellen innerhalb der Zeugenpraxis die spezielle Form der Zeugenschaft eines Fachgutachters oder einer Sachverständigen dar. Lange vor der Neuzeit wurden Chirurgen von den Gerichten zur Wundbegutachtung beigezogen und Hebammen zur Feststellung von Jungfräulichkeit und Schwangerschaft. ${ }^{43}$ Doch auch andere Sachverständige, vor allem Angehörige handwerklicher Berufsgruppen, fungierten beispielsweise in der französischen Rechtspraxis nachweislich seit der Frühen Neuzeit als Zeugen. ${ }^{44}$ Um eine allfällige ständische Befangenheit solcher Fachleute zu verhindern, band man sie 1690 in Paris in permanenten und bezahlten Ämtern an den staatlichen Dienst und machte sie zu offiziel-

40 Kinkelin 1868, 51.

$41 \mathrm{StAB}, \mathrm{BB}$ IV 141, Kontrolle über die vom Regierungsrat genehmigten Gemeinnützigen Gesellschaften 1844-1869.

42 Shapiro 2000, 14.

43 Fischer-Homberger 1983, 31 und 53-68.

44 Hirschi 2013, 12. 
len Experten. ${ }^{45}$ Für die Mediziner begann diese herrschaftliche Bindung noch früher. Im Deutschen Reich übernahmen sie seit dem 16. Jahrhundert Expertenfunktionen als Berater und Beamte und beteiligten sich maßgebend an der Ausarbeitung städtischer Ordnungen. ${ }^{46}$ In Bern ist spätestens ab dem 14. Jahrhundert ein regelmäßig von der Stadt besoldeter Arzt nachgewiesen, dem die Aufsicht über die Apotheker und die Zuständigkeit für ärztliche Gutachten bei Gerichtsverhandlungen übertragen war. ${ }^{47}$ Drohende Epidemien frühzeitig zu erkennen und entsprechende Maßnahmen zu ergreifen fiel in Bern ab 1709 in die Zuständigkeit eines Sanitätsrats, der seinen Aufgabenbereich ständig erweiterte und sich im Laufe des 18. Jahrhunderts zu einer eigentlichen Gesundheitsbehörde medizinischer Sachverständiger entwickelte. ${ }^{48}$

Die ärztlichen Zeugnisse unterscheidet Bloesch in seiner Buchführung von den Gutachten und Berichten, zu denen ihn die Behörden beauftragten. Sein Zeitgenosse Carl Vogel lieferte den Ärzten im staatlichen Dienst die formalrechtliche Unterscheidung zwischen den verschiedenen amtlichen Schriften, zu denen sie angehalten werden konnten, und somit auch diejenige zwischen Gutachten und Zeugnissen. Letztere seien «Aeusserungen unparteiischer Dritter über ihre sinnlichen Wahrnehmungen in Frage stehender That-Sachen», während Erstere vielmehr einen Teil des Richterspruchs darstellten. ${ }^{49}$ Bloesch verfasste also Zeugnisse nicht in der Funktion eines amtlichen Experten, sondern als behandelnder Arzt und Zeuge im Dienst seiner Klientel. Wer bei ihm um ein Zeugnis vorstellig wurde, konnte ganz unterschiedliche Motivationen aufweisen. Zeugnisse wurden einerseits in zunehmendem Maße verwaltungsrechtlich verlangt. Sie konnten aber auch bloß der Absicherung dienen; sie konnten eingeholt werden, um im Falle einer Anklage etwas in der Hand zu haben; sie konnten als gutes Argument in einer außergerichtlichen Streitigkeit dienen; sie konnten dem Schulmeister vorgelegt werden, damit er ein Kind im Unterricht schone.

Mit der Anerkennung der akademischen Ärzte durch die Staatsgewalt, mit der Schließung ihrer Expertenfunktion, gewann ihre professionelle Zeugenschaft an Gewicht, wodurch auch deren Inanspruchnahme durch die Bevölkerung wuchs. Um den Nutzen greifbar zu machen, den die beteiligten Akteure aus den ärztlichen Zeugnissen ziehen konnten, soll nun abschließend das Konzept des medizinischen Marktes beigezogen werden.

45 Vgl. Hirschi 2011, 14.

46 Flügge 2003, 24 und 26.

47 Thurnheer 1944, 16 und 30.

48 Boschung 2008, 295.

49 Vogel 1836, 43 und 49. 


\section{Zeugnisse als Dienstleistung}

Konstitutiv für einen Markt sind Tauschbeziehungen zwischen mehreren Anbietenden und mehreren Nachfragenden. ${ }^{50}$ In der Medizingeschichte hat der Marktbegriff die Perspektive der Forschenden dahingehend verändert, dass sie medizinische Dienstleistungen in ihrer Abhängigkeit von allerhand Konkurrenz auf der einen und von einer wählerischen Nachfrage auf der anderen Seite verstehen mussten.

Das Angebot der ärztlichen Zeugnisse wurde zu Beginn des 19. Jahrhunderts rechtlich stark reguliert, indem nur patentierte Ärzte solche Zeugnisse verfassen durften und in gewissen Fällen nur bestimmte patentierte Ärzte, wie im Falle der Kreisärzte. Diese Regulierung bewirkte erstens, dass bestimmte Verwaltungsabläufe von einer personalen Anwesenheitskommunikation befreit und über die Verschriftlichung formalisiert wurden. ${ }^{51}$ Zweitens hatte sie eine starke Privilegierung der patentierten Ärzte gegenüber deren Konkurrenz zur Folge. Das finanzielle Interesse der Ärzte an den Zeugnissen konnte nicht groß sein. Ein Zeugnis kostete gleich viel wie eine Konsultation und war mindestens so aufwendig. ${ }^{52}$ Doch die Einbindung akademischer Ärzte in staatliche Funktionen bedeutete gleichzeitig den Ausschluss aller nicht-akademischen Heilerinnen und Heiler. Die Ermächtigung, amtliche Zeugnisse verfassen zu dürfen, so die These, bot eine Möglichkeit, das Vertrauen von Patientinnen und Patienten zu gewinnen oder zu halten, ohne durch Heilungserfolge brillieren zu müssen. Aus den Ärzten wurden professionalisierte Zeugen, was sich für sie insbesondere in sozialem Kapital bezahlt machte. Auf der Nachfrageseite sind Interessen und Zwänge schwieriger gegeneinander abzuwägen und hängen vom Einzelfall ab. Einerseits konnten ärztliche Zeugnisse einen großen Vorteil gegenüber jenen Verfahren bedeuten, die persönliche Beziehungen und moralische Unfehlbarkeit voraussetzten. Andererseits ersetzten sie die Möglichkeit des mündlichen Verhandelns durch einen formalistischen Zwang, der durchaus lästig sein und gewisse Abläufe komplizieren konnte. Die staatliche Verwaltung ihrerseits übte eine den medizinischen Markt regulierende Rolle aus, indem sie einerseits die Nachfrage nach ärztlichen Zeugnissen generierte und andererseits die Anbieter dieser Dienstleistung benannte. Als Käuferin ärztlicher Gut-

50 Vgl. z.B. Scherhorn 1989.

51 Zum Konzept der Anwesenheit vgl. Schlögl 2008,155-224.Zur Verschriftlichung vgl. Raphael 2000, 77 und 81-85.

52 Während ein amtliches Gutachten 40 Batzen einbrachte, verlangte Bloesch für ein kurzes Zeugnis 5 Batzen, gleich viel wie für eine Konsultation. Zudem verfasste Bloesch durchschnittlich nur rund 20 Zeugnisse im Jahr. 
achten nahm sie wiederum selbst am Markt teil und festigte die Expertenrolle der Ärzteschaft.

Ulrich Falk spricht in seinen rechtshistorischen Untersuchungen über die juristischen Gutachten der Frühen Neuzeit von einem «Gutachtenmarkt». ${ }^{53}$ Er stellt zudem fest, dass die Auftraggeber von den Gutachtern mit sehr wenigen Ausnahmen begünstigt wurden. Die Gutachter verstanden sich demnach nicht als neutrale Instanz, sondern als Interessenvertreter ihrer zahlenden Klientel und urteilten in deren Interesse, sobald eine gewisse Freiheit in der Normanwendung und -begründung bestand. ${ }^{54}$ Gleiches lässt sich für ärztliche Zeugnisse nicht so leicht feststellen, weil uns der Zugriff auf den Patientenkörper und damit die Entscheidungsgrundlage des Arztes fehlt. Die Interpretationsspielräume waren allerdings in vielen Fällen, vornehmlich bei den Geisteskrankheiten, überaus groß. In einer Kleinstadt wie Biel ist anzunehmen, dass soziale Verflechtungen mindestens gleich wichtig waren wie wissenschaftliche Genauigkeit, wenn es für Bloesch darum ging, sich an die Scharnierstelle zwischen einem Patienten und der staatlichen Verwaltung zu stellen. ${ }^{55}$

\section{Schluss}

Der Übergang von einer medizinischen Policey zur modernen Verwaltung wird zumeist von seinem Resultat her gelesen. Am Ende dieses Prozesses der Ablösung von heterogenem, personalisiertem Wissen stehen vereinheitlichte Ausbildung und Kontrolle der Medizinalpersonen, ärztliches Expertentum sowie ein neuer, öffentlicher Gesundheitsdiskurs. ${ }^{56}$ Diese Geschichte ist vielfach erzählt. Abseits der Modernisierungs- und Medikalisierungsnarrative lenkt das Studium ärztlicher Zeugnisse den Blick auf komplexe Strategien der Aneignung eines neuartigen Machtmittels und auf die Nutzerseite der modernen Verwaltung. Patientinnen und Patienten zeigen sich als selbstbewusste Nutzerinnen und Nutzer eines Angebots, das nicht nur formalisierte soziale Kontrolle bedeutete, sondern ihnen auch gewisse Vorteile brachte. Und Ärzte erscheinen an einem Wendepunkt in ihrem Pro-

53 Vgl. Falk 2006 und Falk 2008.

54 Falk 2006, 389 und 398.

55 Als Beispiel kann der bereits erwähnte Kronenwirt dienen: Am selben Tag, an dem Bloesch erstmals dessen geistige Gesundheit attestierte, stellte er in seiner Anamnese fest, dass Widmer «seit dreien Tagen wieder in sehr aufgeregtem Zustand» sei und «sich wieder mit allerlei fremdartigen Dingen» beschäftige. StadtABiel, 4 A 1, Tagebuch, 16.7.1847, Bd. 30, 130 (Zeugnis) und 132 (Konsultation).

56 Vgl. Sohn 2003, 86. 
fessionalisierungsprozess zum einen als Autoritäten, zum anderen aber auch als Komplizen für ihre Klientel. Wissenschaftlichkeit wird beim Verfassen von Zeugnissen nicht die vorrangige oder einzige Rolle gespielt haben, wenn über die Zeugnisse Klientinnen und Klienten gewonnen wurden, die sich auch anderswo behandeln lassen konnten.

\section{Archivsiglen}

StAB Staatsarchiv Bern

StadtABiel Stadtarchiv Biel

\section{Bibliographie}

Baumer, Matthias, Private und nichtstaatliche Armenfürsorge in der Berner Landgemeinde Worb im 19. Jahrhundert (Nordhausen 2002)

Bloesch, Cäsar Adolf, Bern und Biel. Eine Erinnerung an die grosse Vorzeit (Biel 1853)

Bloesch, Cäsar Adolf, «Allgemeine Grundsätze der theoretischen und der praktischen Medizin, ausgezogen aus meinem Tagbuch ärztlicher Besuche und Konsultationen», in: Mitteilungen der medizinisch-chirurgischen Gesellschaft des Kantons Bern 1 (1866) 1-150

Boschung, Urs, «Medizin und medizinische Versorgung», in: André Holenstein (Hrsg.), Berns goldene Zeit. Das 18. Jahrhundert neu entdeckt (Bern 2008) 294-299

Falk, Ulrich, «Der juristische Gutachtenmarkt. Beobachtungen zum rechtshistorischen Hintergrund», in: Juristische Studiengesellschaft (Hrsg.), Jahresband 2007 (Heidelberg 2008) 129_ 155

Falk, Ulrich, Consilia. Studien zur Praxis der Rechtsgutachten in der frühen Neuzeit (Frankfurt a.M. 2006)

Fangerau, Heiner/Irmgard Müller, «Protokolle des Unsichtbaren: Visa reperta in der gerichtsmedizinischen Praxis des 18. und 19. Jahrhunderts und ihre Rolle als Promotoren pathologischen Wissens», in: Medizinhistorisches Journal 45 (2010) 265-292

Finzsch, Norbert, Obrigkeit und Unterschichten. Zur Geschichte der rheinischen Unterschichten gegen Ende des 18. und zu Beginn des 19. Jahrhunderts (Stuttgart 1990)

Fischer-Homberger, Esther, Medizin vor Gericht. Gerichtsmedizin von der Renaissance bis zur Aufklärung (Bern 1983)

Flückiger Strebel, Erika, «〈Des Standes sanfter Wohlthats-Strom». Staatliche Armenfürsorge auf der Berner Landschaft im 18. Jahrhundert», in: Hans-Jürg Gilomen/Sébastien Guex/Brigitte Studer (Hrsg.), Von der Barmherzigkeit zur Sozialversicherung. Umbrüche und Kontinuität vom Spätmittelalter bis zum 20. Jahrhundert (Zürich 2002) 45-57

Flügge, Sibylla, «RReformation oder erneuerte Ordnung die Gesundheit betreffend». Die Bedeutung des Policeyrechts für die Entwicklung des Medizinalwesens zu Beginn der Frühen Neuzeit», in: Werner Sohn/Bettina Wahrig (Hrsg.), Zwischen Aufklärung, Policey und Verwaltung. Zur Genese des Medizinalwesens 1750-1850 (Wiesbaden 2003)

Frevert, Ute, Die kasernierte Nation. Militärdienst und Zivilgesellschaft in Deutschland (München 2001)

Fröhlich, Sigrid, Die soziale Sicherung bei Zünften und Gesellenverbänden. Darstellung, Analyse, Vergleich (Berlin 1976)

Gesetze, Dekrete und Verordnungen des Kantons Bern (Bern 1805-1848)

Gruner, Erich, Die Arbeiter in der Schweiz im 19. Jahrhundert. Soziale Lage, Organisation, Verhältnis zu Arbeitgeber und Staat (Bern 1968)

Helfer, Monique, Armenwesen und öffentliche Fürsorge der Stadt Biel in der zweiten Hälfte des 19. Jahrhunderts (Lizentiatsarbeit, Bern 1994) 
Hirschi, Caspar, «Moderne Eunuchen? Offizielle Experten im 18. und im 21. Jahrhundert», in: Franz Rexroth (Hrsg.), Wissen, maßgeschneidert. Experten und Expertenkulturen im Europa der Vormoderne (München 2013) (im Druck)

Kinkelin, Hermann, Die gegenseitigen Hülfsgesellschaften im Jahr 1865 (Basel 1868)

Lorenz, Maren, Kriminelle Körper, gestörte Gemüter. Die Normierung des Individuums in Gerichtsmedizin und Psychiatrie der Aufklärung (Hamburg 1999)

Ludi, Regula/Sonja Matter/Tanja Rietmann, «Armut, Armenfürsorge und Sozialpolitik im 19. und 20. Jahrhundert», in: Peter Martig (Hrsg.), Berns moderne Zeit. Das 19. und 20. Jahrhundert neu entdeckt (Bern 2011) 192-204

Ludi, Regula, «Frauenarmut und weibliche Devianz um die Mitte des 19. Jahrhunderts im Kanton Bern», in: Anne-Lise Head/Brigitte Schnegg (Hrsg.), Armut in der Schweiz (Zürich 1989) 19-32

Ludi, Niklaus, Die Armengesetzgebung des Kantons Bern im 19. Jahrhundert. Vom Armengesetz von 1847 zum Armen- und Niederlassungsgesetz von 1897 (Bern 1975)

Meier, Thomas Dominik/Rolf Wolfensberger, «Eine Heimat und doch keine». Heimatlose und Nicht-Sesshafte in der Schweiz, 16.-19. Jahrhundert (Zürich 1998)

Raphael, Lutz, Recht und Ordnung. Herrschaft durch Verwaltung im 19. Jahrhundert (Frankfurt a.M. 2000)

Reusser, Beatrice, Die Krankenversicherung im Kanton Bern im 19. Jahrhundert. Von der Selbsthilfe zur Staatshilfe (Lizentiatsarbeit, Bern 1985)

Scherhorn, Gerhard, «Markt», in: Günter Endruweit/Gisela Trommsdorff (Hrsg.), Wörterbuch der Soziologie (Stuttgart 1989) 416-417

Schläpfer, Rafael, Kantonale Armenreform und kommunale Fürsorgepolitik. Eine Untersuchung über Armenfürsorge im Kanton Bern im 19. Jahrhundert mit dem Schwerpunkt der Einwohnergemeinde Worb (Nordhausen 2002)

Schlögl, Rudolf, «Kommunikation und Vergesellschaftung unter Anwesenden. Formen des Sozialen und ihre Transformation in der Frühen Neuzeit», in: Geschichte und Gesellschaft 2 (2008) 155-224

Shapiro, Barbara J., A Culture of Fact. England 1550-1720 (New York 2000)

Sohn, Werner, «Von der Policey zur Verwaltung: Transformation des Wissens und Veränderungen der Bevölkerungspolitik um 1800», in: Ders./Bettina Wahrig (Hrsg.), Zwischen Aufklärung, Policey und Verwaltung. Zur Genese des Medizinalwesens 1750-1850 (Wiesbaden 2003) $71-89$

Thurnheer, Yvonne, Die Stadtärzte und ihr Amt im alten Bern (Bern 1944)

Vogel, Carl, Das staatsärztliche Verfahren. Für Ärzte, Chirurgen, Apotheker, Thierärzte und für Rechtsgelehrte theoretisch und practisch dargestellt (Jena 1836) 